\title{
A novel method for endoscopic removal of a fractured hydrophilic guide wire from an intrahepatic bile duct
}

Guidewire fracture in which the floppy tip of the wire becomes detached from the shaft is an unusual complication of endoscopic retrograde cholangiopancreatography (ERCP) [1 - 4]. We present here a novel method for endoscopic retrieval of a piece of fractured guide wire from an intrahepatic bile duct.

A 60-year-old man presented at our hospital with a stone in the common bile duct (CBD). Routine manipulation of the sphincterotome and the guide wire (Jagwire, Boston Scientific Corp.) was done in an attempt to achieve the correct orientation for bile duct cannulation during ERCP. Cholangiography showed a CBD stone and a fragment of the guide wire in the distal CBD. After extraction of the stone, the fragment of the guide wire had migrated into the left intrahepatic bile duct. Immediate multiple attempts to remove the fragment in the conventional manner were unsuccessful. At that point, we came up with a novel method. A modified 8.0-Fr dilator was cut about $3 \mathrm{~cm}$ from the end to remove the tapered tip; it was then passed over a new guide wire and advanced into the left intrahepatic duct. Care was taken to ensure that the tip of the dilator was as close as possible as to the fragment of fractured guide wire ( $\bullet$ Fig. 1). The second guide wire was then removed, and $5 \mathrm{ml}$ of normal saline was injected into the left intrahepatic duct through the dilator. Suction with a 20 -ml syringe was then performed through the dilator. The guidewire fragment was successfully aspirated into the channel of the dilator and removed along with it ( Fig. 2 and Fig. 3). The patient recovered and was discharged 3 days later.

This is the first report of successful endoscopic removal of a fragment of fractured guide wire from the bile duct using a modified dilator. This novel technique could be an effective and safe method for removal of small foreign bodies in the biliary tract.

Endoscopy_UCTN_Code_TTT_1AR_2AK

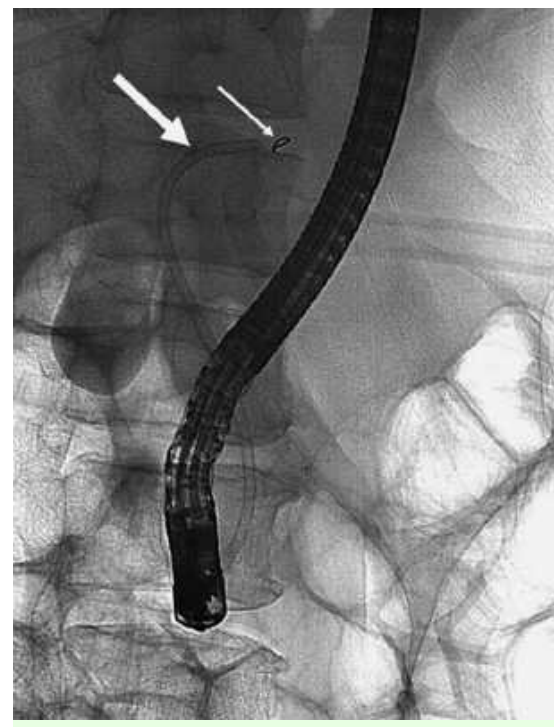

Fig. 1 Radiograph showing the opening tip of the dilator (thick arrow) adjacent to the fragment of the fractured guide wire (small arrow).

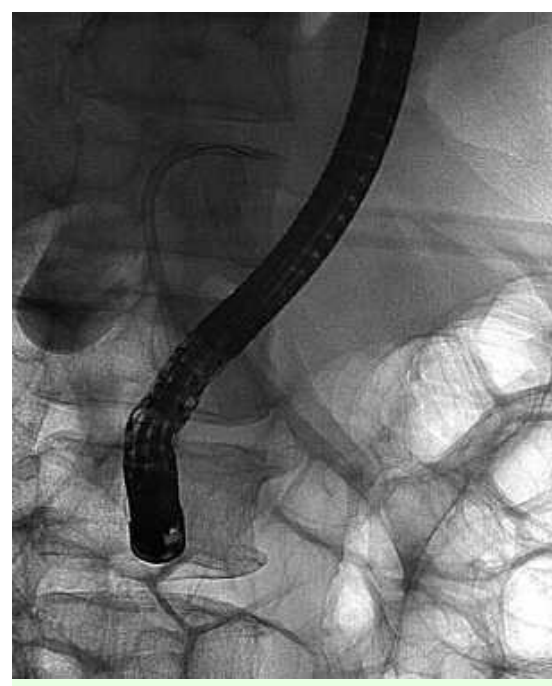

Fig. 2 Radiograph showing the fragment of the fractured guide wire being sucked into the channel of the modified dilator.

F. Liu' ${ }^{1}$, F. Li ${ }^{2}$, Z. D. Jin ${ }^{1}$, D. W. Zou ${ }^{1}$, Z. S. $\mathbf{L i}^{1}$

Department of Gastroenterology, Changhai Hospital, Second Military Medical University, Shanghai, China Division of Gastroenterology and Hepatology, Mayo Clinic Arizona, Scottsdale, Arizona, USA

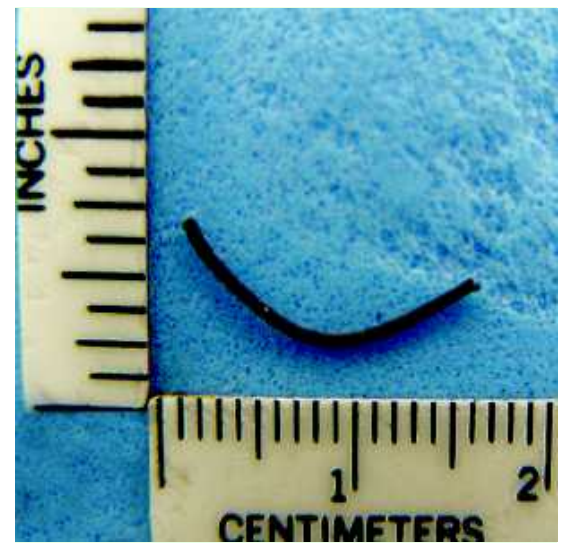

Fig. 3 Fractured floppy end of the hydrophilic guide wire that was removed using the modified dilator.

\section{References}

1 Burdick JS, Schmalz MJ, Geenen JE. Guidewire fracture during endoscopic sphincterotomy. Endoscopy 1993; 25: 251 - 252

2 Heinerman M, Mann R, Boeckl O. An unusual complication in attempted non-surgical treatment of pancreatic bile duct stones. Endoscopy 1993; 25: 248-250

3 Pruitt A, Schutz SM, Baron T et al. Fractured hydrophilic guidewire during ERCP: a case series. Gastrointest Endosc 1998; 48: 77 78

4 Fry LC, Linder JD, Monkemuller KE. Cholangitis as a result of hydrophilic guidewire fracture. Gastrointest Endosc 2002; 56: $943-$ 944

\section{Bibliography}

DOI 10.1055/s-2008-1077662

Endoscopy 2008; 40: E269

(c) Georg Thieme Verlag KG Stuttgart · New York . ISSN 0013-726X

\section{Corresponding author}

\section{Z. S. Li, MD}

Department of Gastroenterology Changhai Hospital

Second Military Medical University

168 Changhai Road

Shanghai

China

Fax: +86-21-55621735

li.zhaoshen@hotmail.com 